Research Article

\title{
Modeling Traffic Function Reliability of Signalized Intersections with Control Delay
}

\author{
Jiayu Hang $(\mathbb{D}$, Xizhao Zhou $(\mathbb{D}$, and Jiawen Wang $\mathbb{D}$ \\ Business School, University of Shanghai for Science and Technology, Shanghai, 200093, China \\ Correspondence should be addressed to Xizhao Zhou; xzzhou@usst.edu.cn
}

Received 27 August 2020; Revised 10 November 2020; Accepted 21 November 2020; Published 9 December 2020

Academic Editor: Luigi Di Sarno

Copyright (C) 2020 Jiayu Hang et al. This is an open access article distributed under the Creative Commons Attribution License, which permits unrestricted use, distribution, and reproduction in any medium, provided the original work is properly cited.

\begin{abstract}
The performance of intersections has been considered a key factor in measuring the efficiency of urban road systems. In this paper, a reliability model for a two-phase signalized intersection is proposed on the basis of presenting a concept of traffic function reliability (TFR). First, classic cumulative curves are created to derive delay formulas. Then, a model for traffic function reliability is proposed based on the quantitative relationship between the random traffic flows, signal timing, and queue lengths. Finally, the delay threshold of the intersection is determined by referring to the level of service. A numerical simulation has been created to clarify the proposed mechanism of TFR. The results show that the saturation and the green time ratio have a dramatical influence on TFR. Under different saturation levels, the sensitivity of TFR to changes in green time ratio gradually weakened. When the green signal ratio increases above a certain value, TFR remains nearly constant. A microscopic simulation verified the applicability of the proposed model. The results show that the accuracy of the model is close to $90 \%$ in the case of low saturation. This method provides road authorities useful insights to understand travel time variability.
\end{abstract}

\section{Introduction}

With the rapid development in communication technologies, passengers, drivers, and traffic managers rapidly obtain real-time traffic information that is used to modify their behavior. Using this real-time information, transportation can evaluate the operational efficiency of urban roads from a random perspective $[1,2]$. Reliability theory has become an effective method to study the stochastic traffic performance [3]. System reliability is defined as the probability that a component or system will perform a required function for a given period of time [4]. Reliability theory provides a more comprehensive insight into the performance of the traffic system and evaluates alternative traffic management strategies. So far, some studies have found that reducing travel time fluctuations can provide travelers with more benefits than just reducing travel time $[5,6]$. Probability distribution is often presented in three different ways:

(1) Histogram. The bar heights in the histogram indicate the relative frequency of a particular condition
(2) Probability Density Function (PDF). PDF portrays the same information as the histogram, except that the bar heights are normalized to 1.0 or $100 \%$

(3) Cumulative Distribution Function. CDF is based on a $\mathrm{PDF}$, where a point value in CDF represents the integral of the PDF up to that instance

Previous studies have analyzed the reliability of networks through topology, which is used to evaluate the efficiency of urban arterials [7]. Existing literature includes research on network connection reliability [8], traffic capacity reliability [9], travel time reliability [10-12], service level reliability [13], and several other aspects [14].

Because it is a significant component, the impact of signalized intersections on network reliability is critical and scholars have carried out research on the reliability at intersections [15]. Delays at signalized intersections account for a significant part of travel time for travelers on urban arterials [16]. Intersection travel time distribution has an important impact on the reliability of networks [5]. Therefore, the concept of intersection reliability has been proposed [17]. 
In this field, some researchers focus on delay distribution models. Olszewski [4, 18] developed an average delay probability distribution model based on a sequential calculation of the queue length probability. Zuylen and Viti [19] modeled the dynamics of the queue length probability distribution within one cycle, rather than using the dynamics of queue length. A generic delay probability distribution expression for both undersaturated and oversaturated situations was derived by assuming the initial queue and stochastic arrivals. However, the expression is rather complicated and may not be well suited for practical applications. Chen et al. [5, 15, 20, 21] performed an analytical model to estimate the distribution of average control delay. The average delay formula is derived through classic cumulative curves. They made an analogy with the Markov chain process to clarify the mechanism of stochastic delays and overflow queues. Based on their analytical model, they estimated the travel time through a convolution of individual link travel time distributions.

Some scholars put forward new performance measures to evaluate signalized intersection reliability. Lo [22] developed a measure to describe the performance of a traffic signal. Their model estimated the probability that an available green time in a given phase is able to clear approaching traffic. They refer to this concept as the phase clearance reliability (PCR). Zhu and Song [23] proposed a concept of travel time versus capacity reliability (TCR) with the consideration of intersection capacity limitation. Combined with probability theory, they analyzed the advantage of the TCR and presented a method to obtain acceptable levels of TCR. To describe whether the start time of signal timing planning adapts to stochastic traffic flow, $\mathrm{Yu}$ et al. [24] put forward the concept of control scheme reliability. They set up a series of virtual experiments using a proposed performance index. The results of their research show that it is effective to optimize signal timing to adapt to the time-varying traffic flow. Using the application of phase clearance reliability (PCR), Lv and Niu [25] explored the quantitative relationship among phase clearance reliability, cycle length, and green time. They deduced the PCR formula in the cases that traffic flow obeys either the normal or negative exponential distribution.

Based on the analysis of previous studies, the two topics are needed. The first is to study the influence of some traffic performance indicators on travel time reliability which includes delays, queue length, and other factors. The other topic uses the intersection signal timing and proposes new indicators such as phase clearance reliability and travel time versus capacity. Research on intersection reliability has received considerable attention. Besides using empirical travel time data, efforts have been made to model travel time reliability through approaches with traffic assignment [26]. However, there has been little discussion about the reliability of real-time signal control intersections. Van Lint et al. classified quantitative indices for travel time reliability into four categories: statistical indices, buffer indices, tardy trip indices, and probabilistic indices. The first three indices are used to measure travel time reliability between OD in the transportation network. The probabilistic indices refer to the proportion of trips whose travel times exceed a predetermined travel time threshold [27]. Thus, the probabilistic index is used to interpret the real-time reliability when vehicles travel through intersections.

The primary objective of this study is to propose a method for signal control intersection reliability and to improve the assessment of these intersections. A proposed TFR model will be used to obtain the reliability of signalized intersections in real time and to provide drivers with information about signalized intersections so they can use that information to plan their routes. This research contributes to a deeper understanding of the intersection travel time reliability. The study makes three contributions:

(1) Using the concept of traffic function reliability (TFR), a method to calculate TFR is created.

(2) The effects of saturation and green time ratio on TFR is studied using a numerical simulation. These results show that under different saturation conditions, the value of TFR gets a rapid rise with an increasing green time ratio. When the green signal ratio increases greater than a certain value, the value of TFR reaches a plateau.

(3) The validity of this model for the case when there is low saturation is verified by a microscopic simulation model after the model was calibrated with actual data.

The structure of this paper is as follows. The first section gives an introduction on quantitative evaluation method of TFR. The second section is concerned with this methodology. In that section, delay formula is created and the TFR model is proposed. Next, the delay threshold is determined at intersections according to the level of service. The last two sections are a numerical case study and a model application, which clarify the proposed mechanism for TFR. The schematic of intersection evaluation is shown in Figure 1.

\section{Problem Description}

2.1. Traffic Function Reliability. The average delay for vehicles is the critical indicator for intersection reliability. In system reliability theory [28], traffic function reliability (TFR) is described as the probability that the average delay reaches the level of traffic function requirements at signalized intersection under specific conditions for a given period of time. A diagram for TFR is shown in Figure 2.

Based on the intelligent transportation system, the reliability of the two-phase signal intersection can be obtained through the TFR model. According to the evolvement of reliability over a period of time, it is possible to reflect the delay variation. As reliability increases, the probability that average delay is less than the delay threshold also increases.

2.2. Parameter Description. The variables that are used in this paper are summarized (Table 1). 


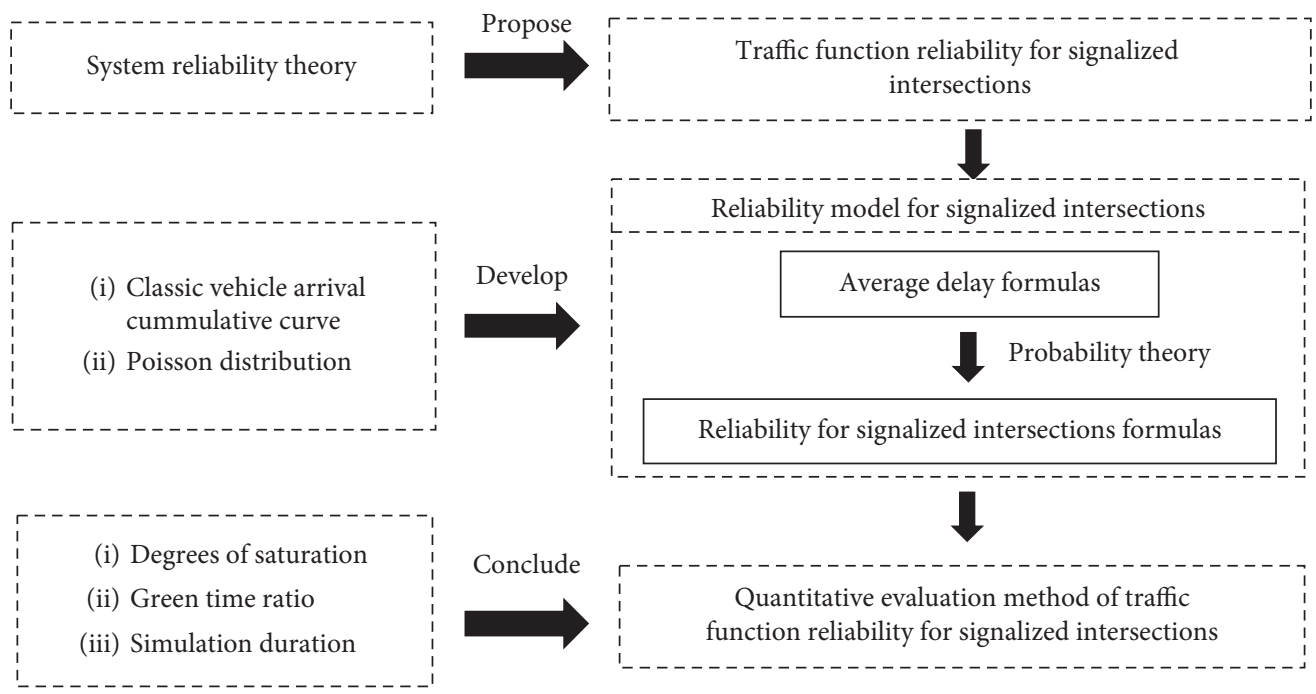

Figure 1: Schematic of intersection evaluation.

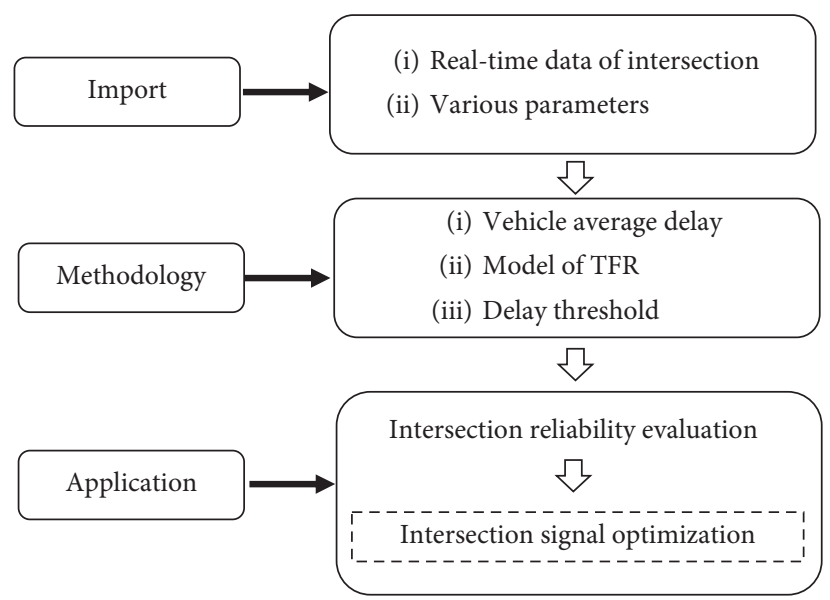

FIgURE 2: Construction layout for TFR.

TABLE 1: Definition of the variables.

$\Phi$ : delay formula

$F$ : probability distribution function

$n_{i}$ : the overflow queue at the beginning of cycle $i$

$q$ : the arrival rate $(\mathrm{veh} / \mathrm{s})$

$s$ : the saturation flow rate (veh/s)

$g_{i}, r_{i}$ : the green time and the red time during cycle $i$ (s)

$\lambda$ : green time ratio

$\bar{d}$ : the average delay $\bar{d}$ for vehicles during cycle $i$ (s)

$t_{c}$ : cycle length (s)

$R_{i}$ : reliability of signalized intersections

$P(\cdot)$ : the probability that indicator attains to the level of traffic

function requirements at signalized intersections

$d_{0}^{\prime}$ : base value of the delay threshold (s)

$\delta$ : adjustment coefficient of delay threshold

$d_{0}$ : delay threshold (s)

$S_{\mathrm{ABEFD}}$ : area of ABEFD

$t^{\prime}:$ the green time when arrival curve and departure curve intersect $v / c$ : ratio of traffic volume to capacity

2.3. Mathematical Description. Figure 3 shows a vehicle queuing diagram. It describes the phenomenon of vehicles queuing in oversaturated situations. The length of vehicle queue reaches the maximum in cycle $i$ when red light phase $r_{i}$ ends. After that, the queue length starts to decrease. When the arrival curve and departure curve intersect, the queue length becomes zero. The vertical dashed lines (Figure 3) represent the queue length, and the gray area is the total delay in cycle $i$ [29].

The delay equations are derived based on the above geometrical relationships. It can be concluded that vehicle average delay is related to signal timing, queue length, traffic flow, and saturation flow. In this paper, it is assumed that the arriving traffic follows a Poisson distribution so that the traffic flow obeys an exponential distribution. The expressions of average delay, traffic flow distribution, and TFR are as follows:

$$
\left\{\begin{array}{l}
\bar{d}=\Phi\left(n_{i}, q, t_{c}, r_{i}, s\right), \\
q \sim E \lambda \\
R_{i}=P\left(\bar{d} \leq d_{o}\right) .
\end{array}\right.
$$

Using equation (1), reliability at a signalized intersection can be described by the following equation: 


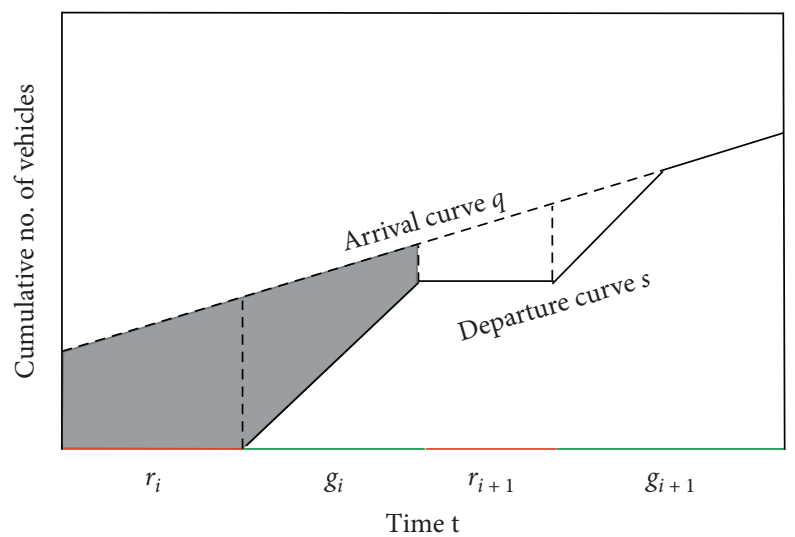

Figure 3: Vehicle queuing.

$$
R_{i}=P\left(\bar{d} \leq t d_{o}\right)=F\left(q=\psi\left(n_{i}, t_{c}, r_{i}, s, d_{o}\right)\right.
$$

\section{Reliability Model}

In this section, the preliminaries are discussed, and then, the estimation method of TFR for a signalized intersection is derived. Afterwards, the method for determining delay threshold is presented.

3.1. Preliminaries. In real life, arrivals are uncorrelated. A common way to deal with this in a model is to assume that the average arrival does not change significantly during the evaluation period. In addition, the arrivals are constant with a specific distribution. For example, Olszewski adopted the Poisson arrival process to model the arrivals evolvement over time at isolated intersections [18]. Later, Zheng et al. analyzed the delay distributions with the Poisson arrival distribution and with the Binomial arrival distribution. Results show that different arrival patterns have little influence on the delay distribution in undersaturated conditions [16].

In this paper, the traffic volume of the intersection of Nanjing Road and Henan Road in Shanghai is collected in an oversaturation condition. The data were collected on May 21, 2018, from $8: 00$ to $8: 45$. By fitting the data, it can be concluded that the traffic volume obeys an exponential distribution and the goodness of fit is shown in Table 2. The Kolmogorov-Smirnov test method is used. The results show that the traffic volume obeys an exponential distribution. Therefore, in this case of oversaturated conditions, it is feasible to assume that the arrival of traffic obeys a Poisson distribution (Figure 4).

As in several previous research studies on travel time variability $[15,20]$, although it is assumed that the number of vehicle arrivals per cycle is a random variable with a known probability distribution, vehicle arrivals are able to be uniformly distributed within each cycle. Intersection handling capacity refers to the maximum number of vehicles that can be dealt with in a given period. It is also regarded as the saturation flow rate $[15,20]$. So far, the saturation flow rate has been assumed to be a constant value [29] or either a binomial [28] or normal [19] distribution.

In this study, TFR for an isolated signalized intersection is estimated in real time. The following is a brief summary of the assumptions that have been made:

(i) The number of vehicle arrivals per cycle is random with a known probability distribution. In the case of an isolated intersection, a Poisson distribution is used.

(ii) The saturation flow rate is assumed to be a constant..

(iii) Traffic function reliability during a certain evaluation period is used as a performance measure of the signalized intersection.

3.2. Reliability Model of a Signalized Intersection. With reference to system reliability theory [28], the concept of traffic function reliability is introduced and defined as the probability that average delay reaches to the level of traffic function requirements at signalized intersections under specific conditions for a given period of time. Mathematically, the traffic function reliability (TFR) is expressed as

$$
R_{i}=P\left(\bar{d} \leq t d_{o}\right)
$$

As shown in equation (3), TFR is coherently related to the average delays. Using real-time data, this paper utilizes the classic vehicle arrival cumulative curve proposed in HCM2000 to construct a calculation model of vehicle delay. The calculation of average delay is divided into two cases (Figure 5).

(1) Figure 5(a) shows that the arrival curve and departure curve do not intersect during cycle $i$, which means that traffic demand exceeds the approach capacity for departures. In that case, not all vehicles could depart during cycle $i$. Some will incur an extra delay waiting for the green phase of cycle $i+1$ or even later cycles. 
TABLE 2: Goodness of fit.

Root mean squared error (RMSE)

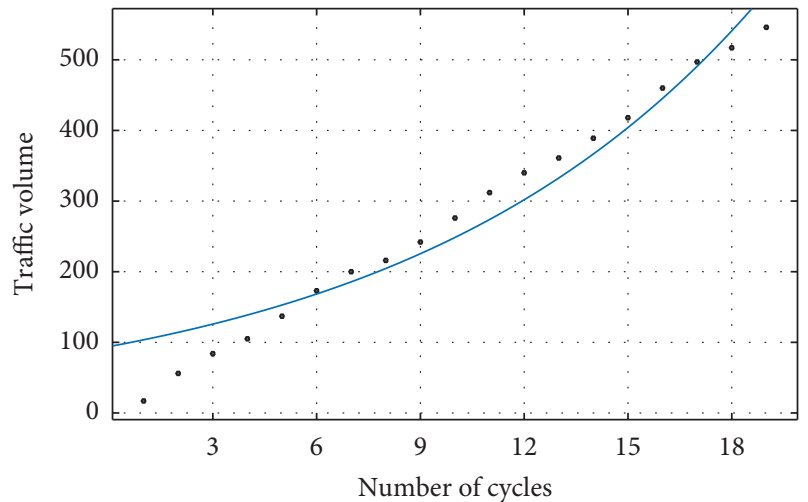

- Volume2 vs. $n$

- Untitled fit 1

Figure 4: Data fitting of traffic volume.

(2) Figure 5(b) shows that the arrival curve intersects the departure curve during cycle $i$. All vehicles are able to depart during the cycle.

According to the geometric relationships described previously, the average delay $\bar{d}$ for vehicles during cycle $i$ is calculated by the following equations:

(1) $n_{i}+q t_{c}>s g_{i}$

$$
\bar{d}=\frac{\left(2 n_{i}+q t_{c}\right) t_{c}-g_{i}^{2} s}{2\left(n_{i}+q t_{c}\right)}
$$

(2) $n_{i}+q t_{c}<s g_{i}$

$$
\bar{d}=\frac{n_{i}^{2}+2 r_{i} s n_{i}+r_{i}^{2} s q}{2(s-q)\left(n_{i}+q t_{c}\right)} .
$$

According to equations (4) and (5), the average delay is related to the overflow queue at the beginning of every cycle and the arrival rate. Therefore, the reliability at signal control intersections can be calculated for four cases:

(1) $n_{i}=0$,

(i) $q t_{c}>s g_{i}$, substitute equation (4) into equation (3).

$$
\begin{aligned}
& R_{i}=F\left(q=\frac{g_{i}^{2} s}{t_{c}^{2}-2 t_{c} d_{o}}\right), \\
& R_{i}=F\left(q=s-\frac{r_{i}^{2} s}{2 t_{c} d_{o}}\right) .
\end{aligned}
$$

(2) $n_{i} \neq 0$,

(i) $n_{i}+q t_{c}>s g_{i}$, substitute equation (4) into equation (3).

$$
R_{i}=\left\{\begin{array}{l}
F\left(q=\frac{g_{i}^{2} s+2 n_{i}\left(d_{0}-t_{c}\right)}{t_{c}^{2}-2 t_{c} d_{0}}\right), \quad t_{c}^{2}-2 t_{c} d_{0} \geq 0, \\
1-F\left(q=\frac{g_{i}^{2} s+2 n_{i}\left(d_{0}-t_{c}\right)}{t_{c}^{2}-2 t_{c} d_{0}}\right), \quad t_{c}^{2}-2 t_{c} d_{0}<0,
\end{array}\right.
$$

$$
R_{i}=P(\varphi(q) \leq 0)
$$$$
\varphi(q)=2 t_{c} d_{0} q^{2}+2 q\left(n_{i} d_{0}+r_{i}^{2} s-s t_{c} d_{0}\right)+n_{i}^{2}+2 n_{i} s\left(r_{i}-d_{0}\right) \text {. }
$$

(ii) $q t_{c} \leq s g_{i}$, substitute equation (5) into equation (3).

(ii) $n_{i}+q t_{c} \leq s g_{i}$, substitute equation (5) into equation (3).

Note that the signalized intersection system is governed by a cyclic mechanism [30]. This method allows researchers to use discrete time steps, which are equal to the cycle length, and to calculate the initial queue length distribution at the beginning of every period. Regardless of the timing control or real-time adaptive control strategy, the state of $n_{i+1}$ depends only on the state of the previous $n_{i}$ and the number of arrivals and departures in a period of time. This method is also referred to as renewal theory [31], where $n_{i+1}$ can be calculated by the following equation:

$$
n_{i+1}=n_{i}+q t_{c}-s g_{i}
$$

\section{Delay Threshold}

The quantity, $d_{0}$, in equation (3) represents the delay threshold. TFR represents the probability that the average delay is less than $d_{0}$. In HCM2000, control delay refers to the extra time experienced by users due to the signal controllers, including the time of vehicle acceleration, deceleration, and waiting for the green light [29]. According to the code for design of urban road engineering of China (CJJ37-2012) [32], the level of service at urban road intersections is divided into four regions (Table 3 ).

In this study, control delay of intersection in the first level of service is used as the basis for determining the delay threshold. Different delays result from the mix of vehicle speed, intersection level of service, and intersection location. Accordingly, taking these interfering factors into consideration is necessary.

When in commercial areas, the travel time is more valuable; correspondingly, the delay threshold should be smaller than in suburbs. As a whole, delay increases as the 


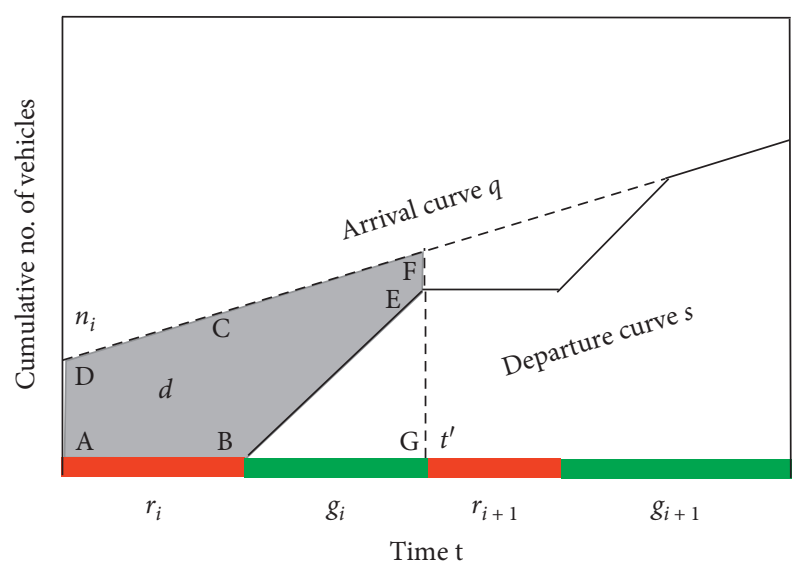

(a)

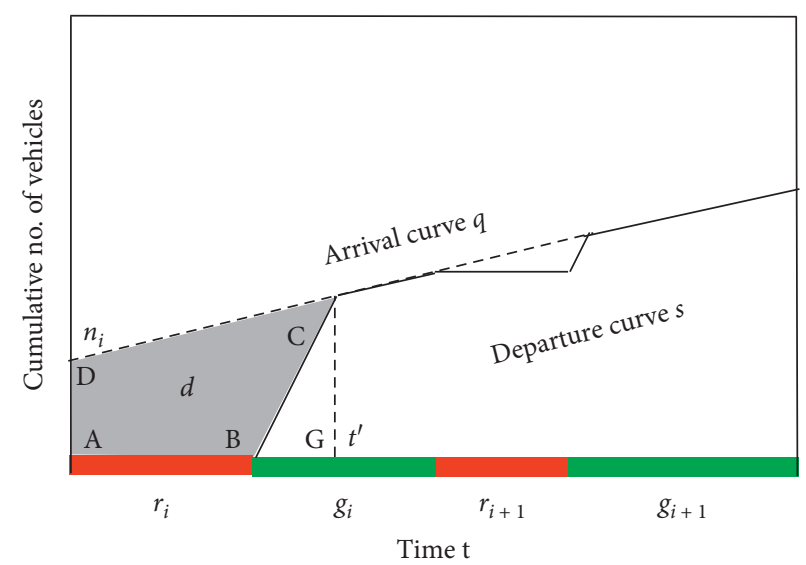

(b)

FIgURE 5: Delay in cycle $i$ : (a) arrival traffic demand is higher than approach capacity. (b) Arrival traffic demand is lower than approach capacity.

TABLE 3: Signal control intersections service level.

\begin{tabular}{lcccc}
\hline Level of service & 1 & 2 & 3 & 4 \\
\hline Control delay $\left(\mathrm{s} / \mathrm{vel}^{-1}\right)$ & $<30$ & $30 \sim 50$ & $50 \sim 60$ & $\geq 60$ \\
$v / c$ rate & $<0.6$ & $0.6 \sim 0.8$ & $0.8 \sim 0.9$ & $\geq 0.9$ \\
Queue length $(\mathrm{m})$ & $<30$ & $30 \sim 80$ & $80 \sim 100$ & $\geq 100$ \\
\hline
\end{tabular}

number of phases increases. In order to unify the evaluation criteria, the delay threshold with a large number of phases should be larger. Taking the above influencing factors into account, this paper introduces the adjustment coefficient $\delta$ to determine the delay threshold. The traffic environment of each intersection is different and traffic conditions of the same intersection can also be different in different time periods, which should be considered at the same time when determining the delay threshold. Therefore, the range of adjustment coefficient $\delta$ is given alone. For each actual intersection, the $\delta$ will be obtained after sensitivity analysis in different conditions. The range of adjustment coefficient $\delta$ is defined in equation (11) and the values are shown in Table 4.

$$
d_{0}=\delta \times d_{0}^{\prime} .
$$

\section{Numerical Examples}

In this section, saturation and green time ratio are taken into account to investigate their effect on the TFR. To illustrate the applicability of the proposed models, this study applies the control approach to an intersection, which has two lanes (one approaching lane and one exit lane) at each arm (Figure 6). The simulation time is 30 minutes and the initial queue is zero at the beginning of each simulation. The parameters are shown in Table 5.

5.1. Sensitivity to Different Degrees of Saturation. This section will analyze the evolution of TFR and delay probability distribution for saturation values of $0.5,0.7$, and 0.9 . The results show that when 30 cycles are simulated, the TFR of the intersection tends to be stable. Consequently, this paper using an evaluation period is 30 cycles.

Figure 7 illustrates the evolution of the average delay for different saturation cases $(v / c=0.5$ to 0.9$)$. In this region, the shape of delay distribution gradually evolves from a single peak to a bimodal distribution. The figure further shows that the average delay distribution is significantly influenced by the saturation.

Figures $8(\mathrm{a})$ and $8(\mathrm{~b})$ show the evolution of average delay and TFR for a saturation of 0.5. It is apparent that the shape of the delay distribution changes very little after the first five cycles. The average delay probability exhibits a unimodal distribution over time (Figure 8(a)). TFR is approximately 0.7 as the number of cycles increases (Figure $8(b)$ ). When traffic demand is less than the saturated flow, almost all vehicles can depart in one cycle. In that case, the TFR at the intersection is high. Therefore, the results of numerical simulation are consistent with the actual situation.

Figures $8(\mathrm{c})$ and $8(\mathrm{~d})$ illustrate the evolution of average delay and TFR at a saturation of 0.7. As the number of cycles increases, the probability of average delay gradually changes from a single peak to a bimodal distribution (Figure 8(c)). From Figure 8(d), we can see that when the saturation is 0.7 , the value of TFR eventually stabilizes around 0.4 . With a saturation of 0.5 , the TDR decreases to 0.3 . A possible explanation for these results might be that the later arrivals in a cycle are prone to be influenced by overflow queues and therefore must wait for more than one cycle. Consequently, the probability of a high delay increases.

Figures $8(\mathrm{e})$ and $8(\mathrm{f})$ describe the evolution of the average delay and TFR under a saturation of 0.9 . The rapid change of delay distribution becomes quite apparent (Figure 8(e)). Compared to the saturation at 0.7 , the probability of vehicles waiting for more than one cycle increases faster, and the probability of high delay is greater. When the traffic flow is close to saturation, the TFR is as low at about 0.1 (Figure 8(f)). As the flow saturation increases, vehicles entering the 
TABLE 4: Range of adjustment coefficient $\delta$.

\begin{tabular}{lccc}
\hline Phase & & Control area & \\
& Intersections in commercial district & Intersections in residential area & Intersections in fringe area \\
\hline Two-phase & $(0.90,1.00]$ & $(1.00,1.10]$ & $(1.10,1.20]$ \\
Three-phase & $(0.95,1.05]$ & $(1.05,1.15]$ & $(1.15,1.25]$ \\
Four-phase & $(1.00,1.10]$ & $(1.10,1.20]$ & $(1.20,1.30]$ \\
\hline
\end{tabular}

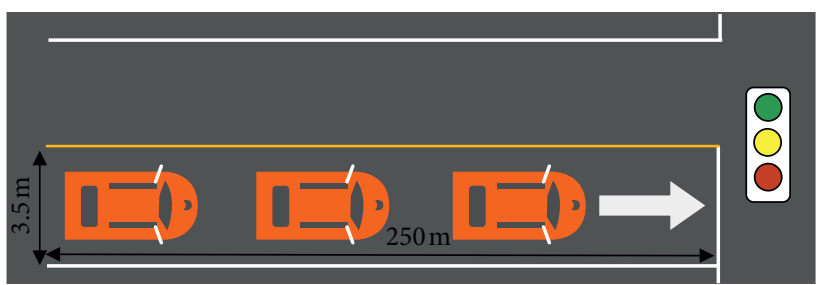

Figure 6: Geometric layout of an arm in the numerical case.

TABle 5: Parameters in numerical case study.

\begin{tabular}{lcccc}
\hline Cycle length, $t_{c}(\mathrm{~s})$ & Green time, $g_{i}(\mathrm{~s})$ & Red time, $r_{i}(\mathrm{~s})$ & Saturation flow rate, $s(\mathrm{pcu} / \mathrm{h})$ & Delay threshold $(\mathrm{s})$ \\
\hline 60 & 24 & 36 & 1800 & 30 \\
\hline
\end{tabular}

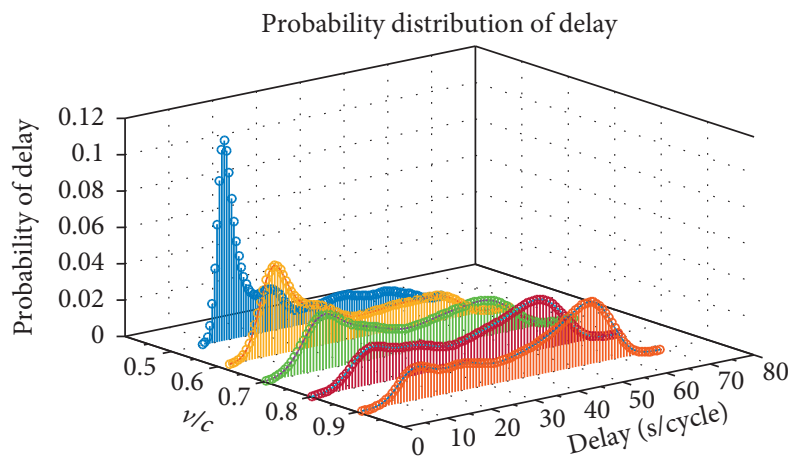

FIgURE 7: The evolution of delay probability distribution under the degrees of saturation from 0.5 to 0.9 .

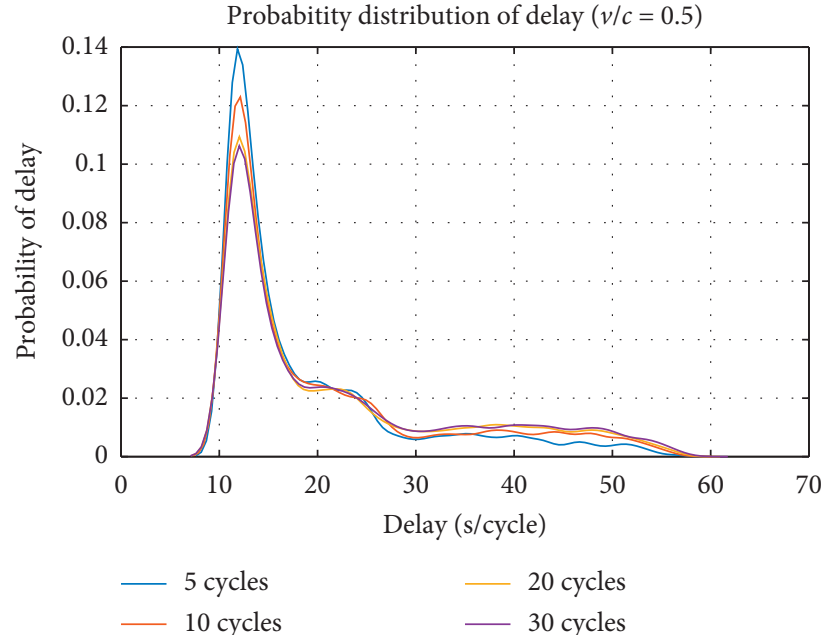

(a)

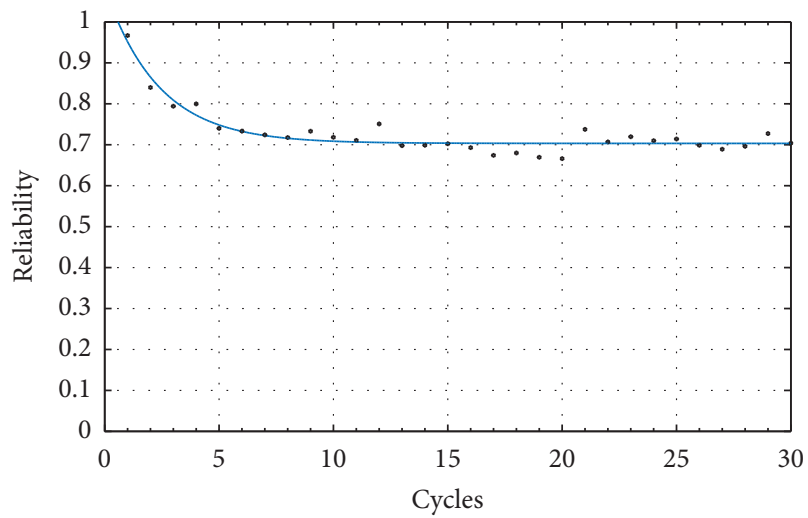

(b)

FIGURE 8: Continued. 


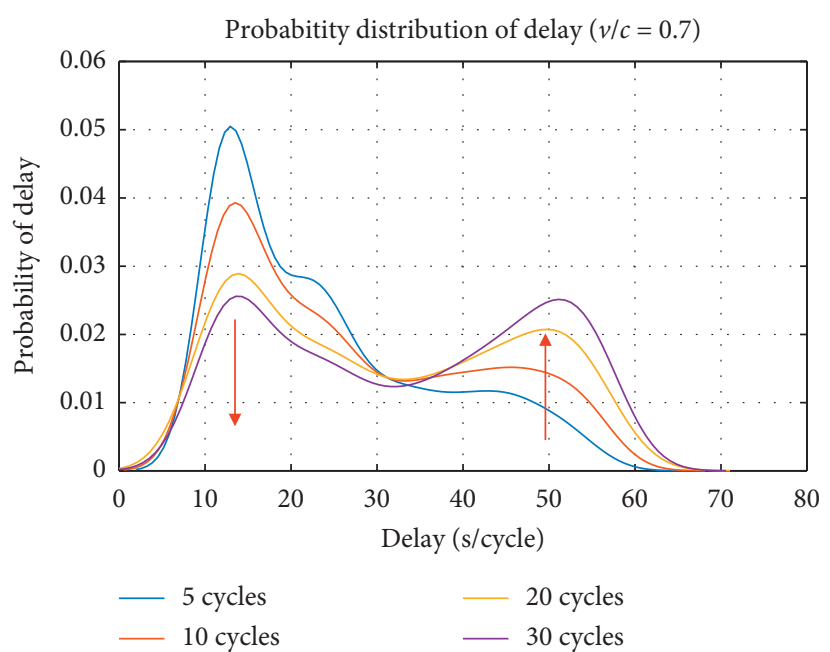

(c)

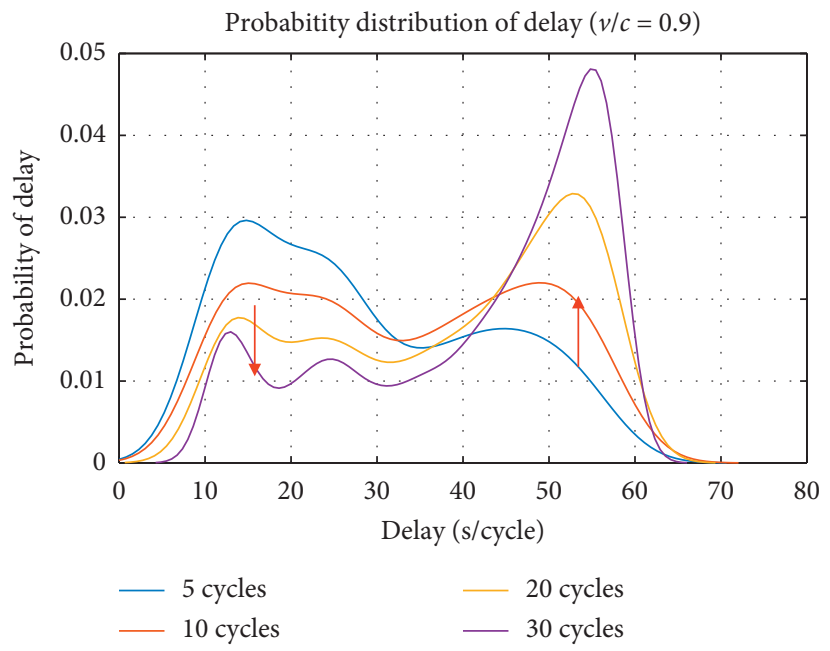

(e)

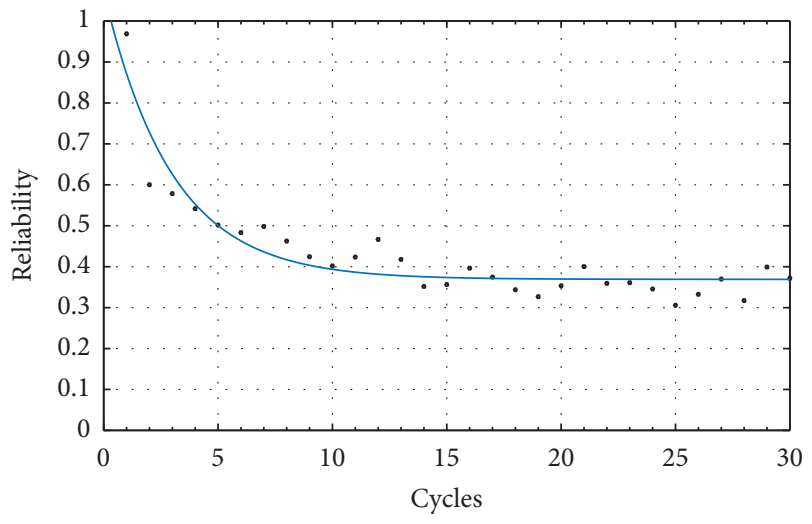

(d)

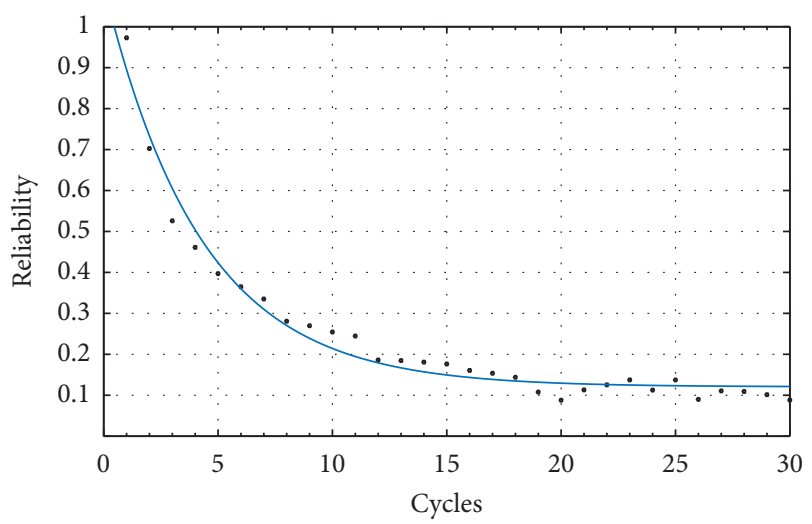

(f)

Figure 8: Delay distribution and reliability degree $(v / c=0.5,0.7$, and 0.9$)$. (a) Delay distribution. (b) Evolution of TFR. (c) Delay distribution. (d) Evolution of TFR. (e) Delay distribution. (f) Evolution of TFR.

intersection approach queue up. In this case, the driving behavior becomes more complicated, and the traffic capacity changes. Therefore, it is possible that the TFR of 0.1 under high saturation may be inconsistent with real conditions.

According to the above analysis, it can be concluded that the intersection average delay changes from a unimodal distribution to a bimodal distribution with an increase of traffic flow saturation, and the intersection reliability decreases significantly with the increase of traffic flow. In low flow saturation, the TFR of a signalized intersection is high and most vehicles can depart the intersection in one signal cycle. While in the high flow saturation condition, the TFR becomes low and the probability of high vehicle delay is increased. In this case, the traffic efficiency at intersections is reduced.

5.2. Sensitivity to Different Green Time Ratio. The analysis in Section 4.1 shows the influence of saturation variation on delay probability distribution and TFR. In this section, the evolution of TFR with the change of green time ratio is analyzed.
Figure 9 describes the evolution of TFR at a signal control intersection approach under the saturation of 0.3 , 0.5 , and 0.7. The cycle length is $60 \mathrm{~s}$. What stands out in this figure is that the green time ratio has a significant impact on TFR. For low values of the green time ratio, TFR increases rapidly, while it becomes constant for higher values of the green time ratio. What is surprising is that when the TFR becomes a constant at different saturation value, the green time ratio is still less than 1 . This result is indicated in the red box area. These results indicate that the adjustment of the signal timing plan does not necessarily improve the intersection reliability. Changes in TFR are more sensitive at low saturation. When the green time ratio is constant, overall, the TFR is higher than that at high saturation (Figure 9).

5.3. Comparison between Numerical Analysis and Real-World Example. The traffic data and signal control scheme of two actual intersections (the intersection of Boshi Road and Zuchongzhi Road; the intersection of Boshi Road and 


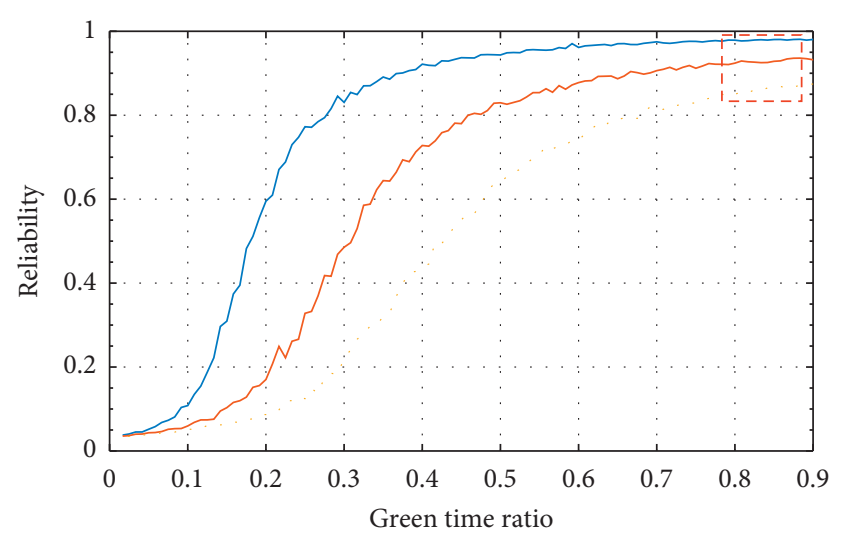

$V / C$ :

$-0.3$

$-0.5$

$-0.7$

Figure 9: Green time ratio: the reliability of the signalized intersection approach.

Guanlin Road) are collected to analysis the ability of the proposed model to solve real-world problems. According to the actual data obtained, the reliability of the two intersections is calculated, respectively, whose results are compared with those in the sensitivity analysis, as shown in Figures 10 and 11 .

As shown in Figure 10, according to the traffic data during the morning peak period, the TFR of the intersection of Boshi Road and Zuchongzhi Road is obtained based on the proposed model. The saturation in morning peak period is similar to 0.9. Hence, the TFR of actual intersection is compared with the TFR at a saturation of 0.9 in the numerical analysis. It is apparent that the traffic function reliability is low and the probability that average vehicle delay is larger than delay threshold is high due to the high saturation degree, which is consistent with the actual situation.

As shown in Figure 11, the TFR of the intersection of Boshi Road and Guanlin Road is obtained during the offpeak period. The saturation is about 0.3. The TFR is compared with that at the saturation of 0.5 in numerical analysis. It is obvious that the reliability of actual intersection is higher than that in the saturation of 0.5. This result indicates that when the traffic flow saturation is lower, the reliability of the intersection is higher, and the probability that the average vehicle delay is less than the delay threshold is higher.

\section{Model Validation}

To verify the applicability of the proposed method, this section constructs a case intersection, which was microscopically simulated at saturations of $0.3,0.5$, and 0.7 . The case intersection is a two-phase signal control intersection that is illustrated in Figure 12. The parameters of the intersection are shown in Table 6.

The vehicle speed and vehicle composition are the default values. The green time ratio $\lambda$ changes from 0.1 to 0.9

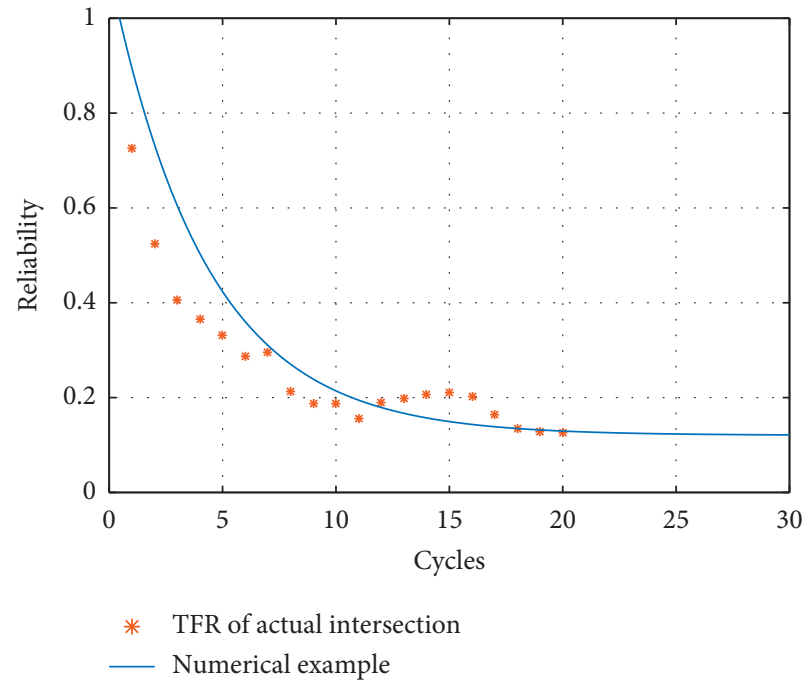

FIgURE 10: Traffic function reliability of actual intersection and in numerical example with a saturation of 0.9 .

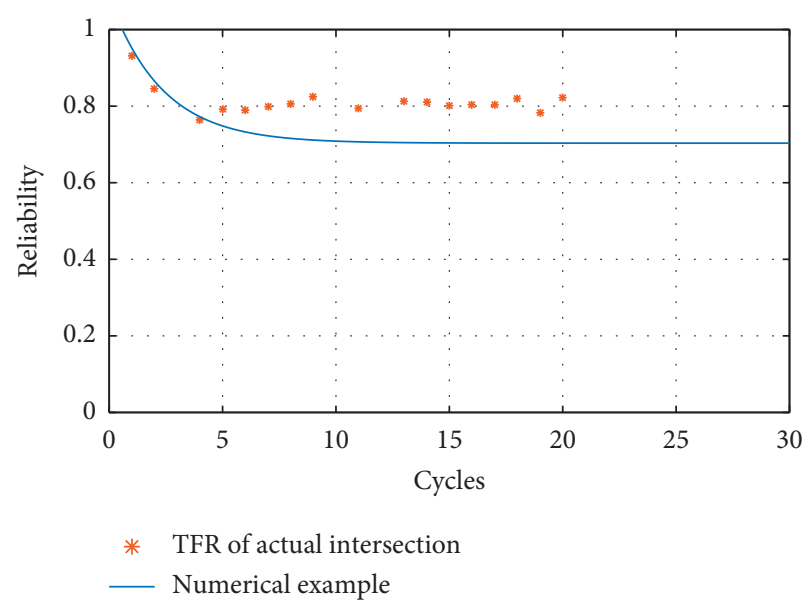

FIgURE 11: Traffic function reliability of actual intersection for a saturation of 0.3 and in numerical example with a saturation of 0.5 .

with a 0.05 step size. The simulation runs for $3600 \mathrm{~s}$ under every signal timing plan, and random seeds are different for each simulation. When the green time ratio is low (e.g., $\lambda=0.1,0.15,0.2,0.25,0.3$, and 0.35), a warning appears during the simulation, and some vehicles do not pass through the intersection at the end of simulation. In this case, only the data of vehicles leaving the intersection are selected for the analysis. After the microsimulation, the TFR of westward approach was calculated. The comparison between microscopic simulation and model estimation is shown in Figure 13.

Figures 13(a)-13(c) present a comparison between microsimulation and model estimation. At a saturation of 0.3 , TFR increases rapidly as $\lambda$ increases. When $\lambda$ is greater than 0.5 , the TFR of the west approach tends to be stable. A possible explanation for this phenomenon is that in the case of low saturation, almost all arrivals are able to leave in one cycle. Therefore, most vehicles experience low delays and the 


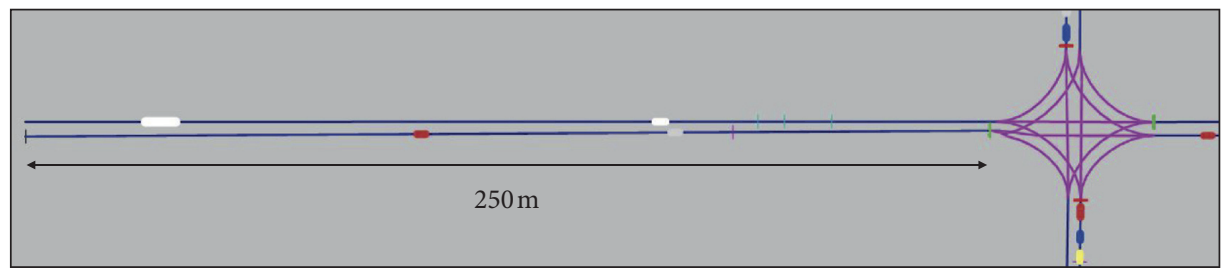

FIGURE 12: Geometric layout of an arm in the microsimulation.

TABle 6: Parameters in microscopic simulation.

\begin{tabular}{lccccc}
\hline Cycle length, $t_{c}(\mathrm{~s})$ & Lanes & Lane width $(\mathrm{m})$ & Lane length $(\mathrm{m})$ & Simulation time $(\mathrm{s})$ & Delay threshold $(\mathrm{s})$ \\
\hline 60 & 2 & 3.5 & 250 & 3600 & 30 \\
\hline
\end{tabular}

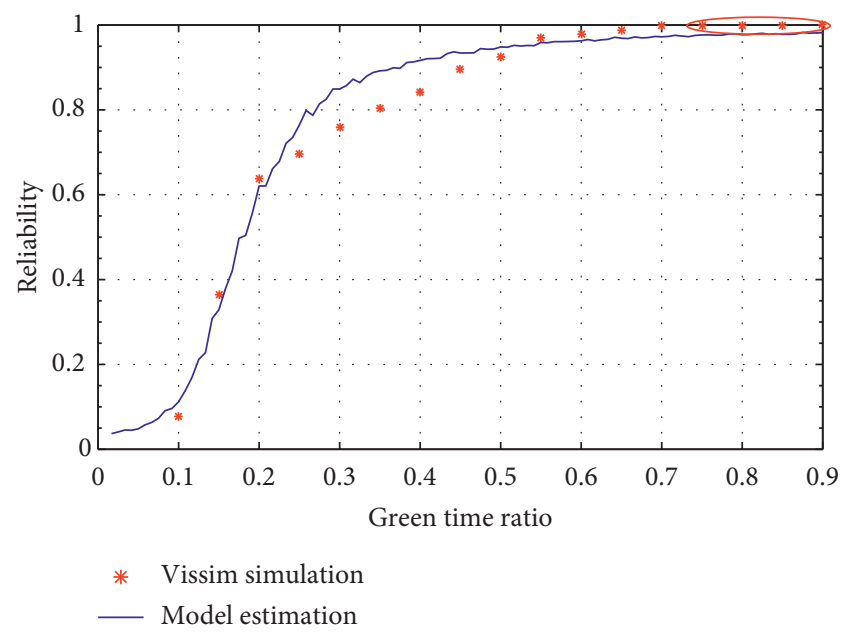

(a)

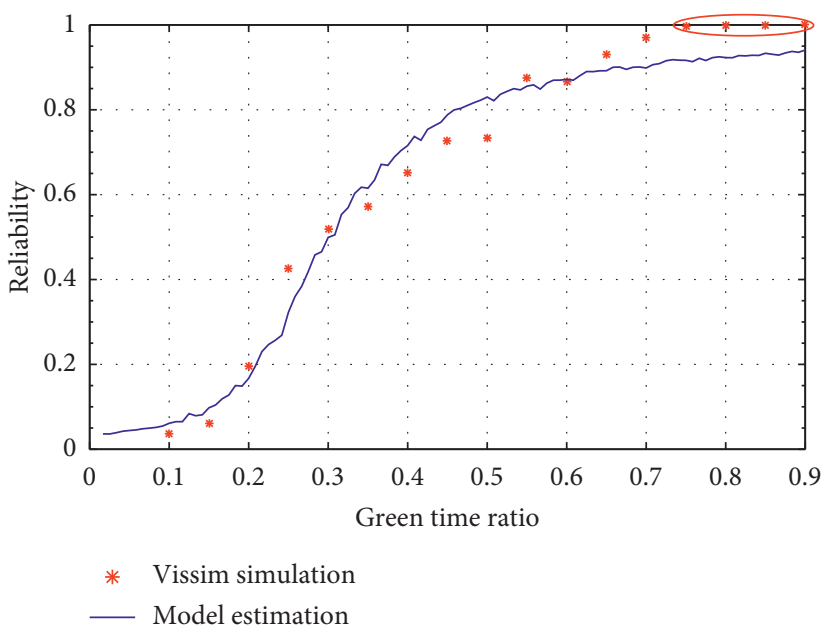

(b)

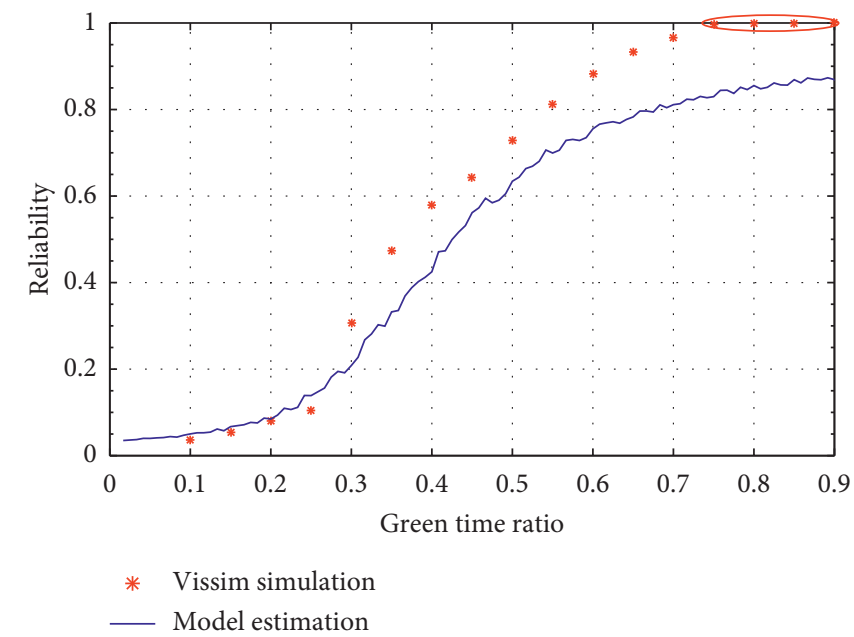

(c)

FIgURE 13: A comparison between microsimulation calculation and model estimation. (a) $v / c=0.3$, (b) $v / c=0.5$, and $(c) v / c=0.7$.

TFR rapidly tends to 1 . At a saturation of 0.5 and 0.7 , the TFR gradually reaches 1 when $\lambda$ is more than 0.75 . When the saturation is high, the TFR can be increased to 1 only by obtaining a longer green time. It is somewhat surprising that the green time ratio is far less than 1 when the TFR reaches 1 , as shown in the red marker (Figure 13).
Figures 14 and 15 provide the deviation between microsimulation calculation and model estimation. It can be seen that the absolute error of approach reliability is less than 0.2 overall. When the saturation is 0.3 , the absolute error between microsimulation and model estimation is less than 0.1 . The relative error is less than $20 \%$, 


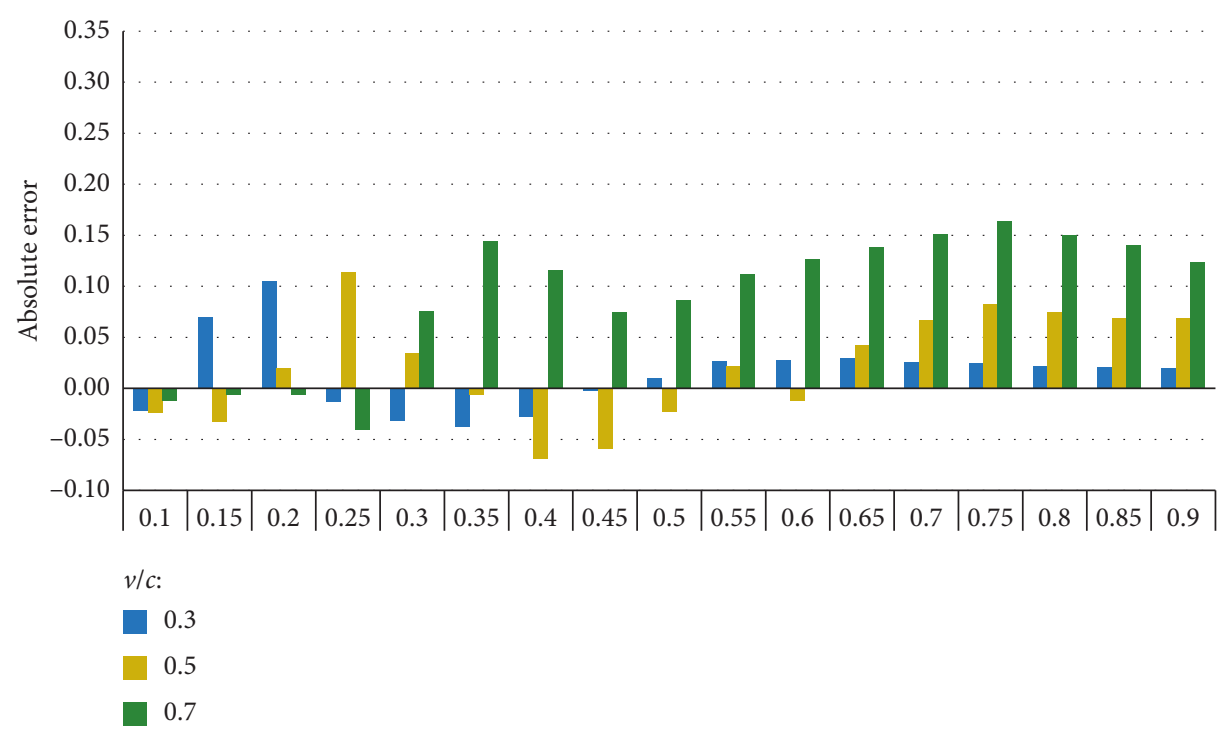

FIGURE 14: Mathematical deviation between microsimulation calculation and model estimation.

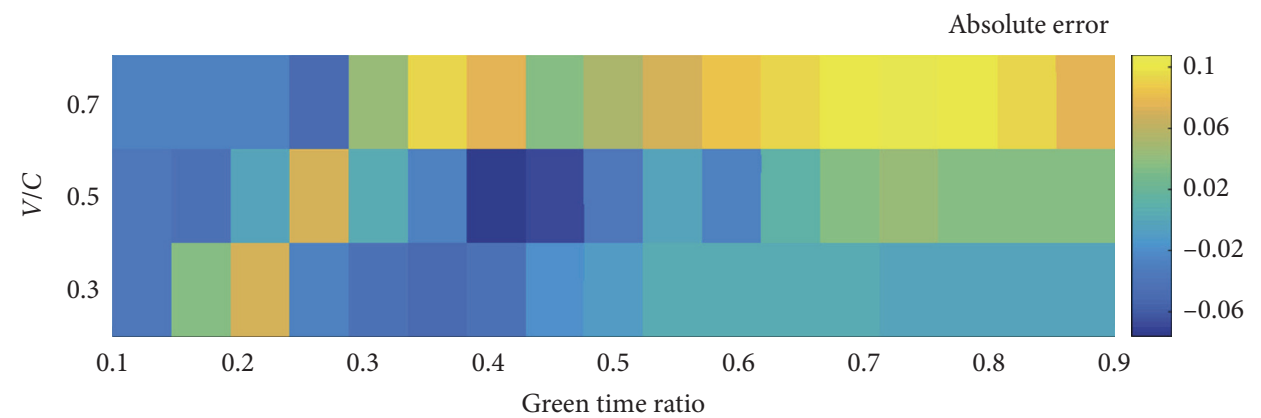

(a)

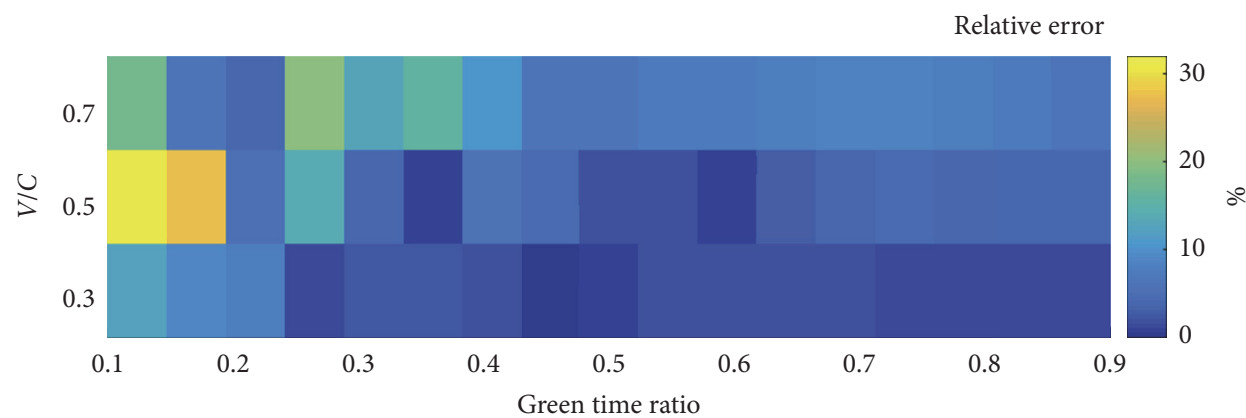

(b)

FIgURE 15: Absolute error and relative error between microsimulation calculation and model estimation.

except when the green time ratio is 0.1 . When the flow rate is in the case of high saturation, the absolute error is all most more than 5\%. Especially in the flow rate of 0.7 , the absolute error is more than $10 \%$, even though in the situation of high green time ratio. A comparison of the two results reveals it is reasonable to evaluate the operation of signalized intersections using the reliability model constructed. At the same time, these results further support the assumption that this paper's model is more suitable to the reliability evaluation under low and medium traffic saturation.

\section{Conclusions}

This study proposes a TFR model to evaluate the reliability of signal control intersections. The model introduces traffic function reliability on the basis of probability theory. A procedure was proposed for calculating TFR considering an average delay. After that, the numerical case study of TFR was conducted, taking into various factors (i.e., saturation and green time ratio). The model was evaluated in microsimulation, and the results indicate the ability of the proposed method to assess the reliability of intersections. It can 
be seen from the results that the accuracy of the model solution is close to $90 \%$ in the case of low saturation, which indicates that the proposed model is reasonable in evaluating the reliability of intersections.

In summary, the ability to capture the evolution of the TFR at signal control intersections is a critical step in evaluating the performance of urban roads. Gaining knowledge of intersection reliability makes it possible to estimate the probability of the average delay. However, it is unfortunate that this study only considered the reliability of isolated intersections and did not consider the impact of adjacent intersections. One should increase the complexity of this model by studying the reliability of correlated intersections. Additionally, the focus on identifying the entire intersection reliability is beneficial to the evaluation of urban road system. This will be the foundation of our future work.

\section{Data Availability}

All the data used to support the findings of this study are included within the article.

\section{Disclosure}

The content of this paper reflects the views of the authors, who are responsible for the facts and the accuracy of the data presented herein.

\section{Conflicts of Interest}

The authors declare that they have no conflicts of interest.

\section{Acknowledgments}

The authors thank the National Natural Science Foundation of China Project "Research on key issues of reliability analysis for random traffic network travel time" (61273042) for the support.

\section{References}

[1] J. Wang, Y. H. Kim, X. Z. He, and S. Peeta, "Analytical model for information flow propagation wave under an information relay control strategy in a congested vehicle-to-vehicle communication environment," Transportation Research Part C: Emerging Technologies, vol. 94, pp. 1-18, 2018.

[2] H. Li, F. He, X. Wang, and X. Lin, "Travel time reliability measure based on predictability using the Lempel-Ziv algorithm," Transportation Research Part C: Emerging Technologies, vol. 101, pp. 161-180, 2019.

[3] X. Chen, C. Osorio, and B. F. Santos, "Simulation-based travel time reliable signal control," Transportation Science, vol. 53, no. 2, pp. 523-544, 2019.

[4] M. S. Hossan, H. Asgari, and X. Jin, "Investigating preference heterogeneity in value of time (VOT) and value of reliability (VOR) estimation for managed lanes," Transportation Research Part A: Policy and Practice, vol. 94, pp. 638-649, 2016.

[5] P. Chen, J. Sun, and H. Qi, "Estimation of delay variability at signalized intersections for urban arterial performance evaluation," Journal of Intelligent Transportation Systems, vol. 21, no. 2, pp. 94-110, 2017.
[6] F. Zheng, H. J. Van Zuylen, X. Liu, and S. Le Vine, "Reliabilitybased traffic signal control for urban arterial roads," IEEE Transactions on Intelligent Transportation Systems, vol. 18, no. 3, pp. 643-655, 2017.

[7] C. Sun, L. Cheng, S. Zhu, F. Han, and Z. Chu, "Multi-criteria user equilibrium model considering travel time, travel time reliability and distance," Transportation Research Part D: Transport and Environment, vol. 66, pp. 3-12, 2017.

[8] Y.-S. Qian, M. Wang, H.-X. Kang, J.-W. Zeng, and Y.-F. Liu, "Study on the road network connectivity reliability of valley city based on complex network," Mathematical Problems in Engineering, vol. 2012, pp. 1-14, Article ID 430785, 2012.

[9] J. Wang and W. Deng, "Optimizing capacity of signalized road network with reversible lanes," Transport, vol. 33, no. 1, pp. 1-11, 2018.

[10] S. C. Wong and H. Yang, "Reserve capacity of a signal-controlled road network," Transportation Research Part B: Methodological, vol. 31, no. 5, pp. 397-402, 1997.

[11] F. Fu, D. Ma, D. Wang, and W. Qian, "An optimization method of time window based on travel time and reliability," Mathematical Problems in Engineering, vol. 2015, Article ID 921480, 9 pages, 2015.

[12] M. K. H. Shehada and A. Kondyli, "Evaluation of ramp metering impacts on travel time reliability and traffic operations through simulation," Journal of Advanced Transportation, vol. 2019, Article ID 8740158, 2019.

[13] J. Wang, C. Wang, J. Lv, Z. Zhang, and C. Li, "Modeling travel time reliability of road network considering connected vehicle guidance characteristics indexes," Journal of Advanced Transportation, vol. 2017, Article ID 2415312, 9 pages, 2017.

[14] X. F. Zhang, Q. Zhong, and Q. Luo, "Evaluation of transportation network reliability under emergency based on reserve capacity," Journal of Advanced Transportation, vol. 2019, Article ID 1370159, 2019.

[15] P. Chen, F. Zheng, G. Lu, and Y. Wang, "Comparison of variability of individual vehicle delay and average control delay at signalized intersections," Transportation Research Record: Journal of the Transportation Research Board, vol. 2553, no. 1, pp. 128-137, 2016.

[16] F. F. Zheng and H. V. Zuylen, "Uncertainty and predictability of urban link travel time delay distribution-based analysis," Transportation Research Record Journal of the Transportation Research Board, vol. 2192, no. 1, pp. 136-146, 2010.

[17] W. X. Qiao and D. Wang, "A transit signal priority optimizing model based on reliability," Journal of Transportation Systems Engineering and Information Technology, vol. 17, no. 2, pp. 54-59, 2017.

[18] P. S. Olszewski, "Traffic signal delay model for non-uniform arrivals," Transportation Research Record, vol. 1287, pp. 4253, 1990.

[19] H. V. Zuylen and F. Viti, "Probabilistic model for queues, delays, and waiting time at controlled intersections," in 86th Meeting of the Transportation Research Board, pp. 21-25, Washington, DC, USA, January 2007.

[20] P. Chen, H. Liu, H.-S. Qi, and F.-J. Wang, "Analysis of delay variability at isolated signalized intersections," Journal of Zhejiang University SCIENCE A, vol. 14, no. 10, pp. 691-704, 2013.

[21] M. Chen, G. Yu, P. Chen, and Y. Wang, "A copula-based approach for estimating the travel time reliability of urban arterial," Transportation Research Part C: Emerging Technologies, vol. 82, pp. 1-23, 2017. 
[22] H. K. Lo, "A reliability framework for traffic signal control," IEEE Transactions on Intelligent Transportation Systems, vol. 7, no. 2, pp. 250-260, 2006.

[23] Y. B. Zhu and C. Song, "Reliability analysis of urban plane intersection," Journal of Transport Information and Safety, vol. 28, no. 1, pp. 31-34, 2010.

[24] Q. Yu, S. Dai, and F. T. Ren, "Control scheme reliability research for signalized intersection," Journal of Beijing University of Technology, vol. 33, no. 10, pp. 1066-1069, 2007.

[25] B. Lv and H. Niu, "Reliability modeling and simulation of signalized intersections," Journal of Transportation Systems Engineering and Information Technology, vol. 11, no. 6, pp. 45-50, 2011.

[26] Y. Gu, X. Fu, Z. Y. Liu, X. Xu, and A. Chen, "Performance of transportation network under perturbations: reliability, vulnerability, and resilience," Transportation Research Part E-Logistics and Transportation Review, vol. 133, pp. 1-16, 2020.

[27] J. W. C. Van Lint, H. J. Van Zuylen, and H. Tu, "Travel time unreliability on freeways: why measures based on variance tell only half the story," Transportation Research Part A: Policy and Practice, vol. 42, no. 1, pp. 258-277, 2008.

[28] Y. D. Yan, P. Tong, Z. Q. Ning, and D. W. Wang, "Quantitative evaluation method of traffic function reliability for urban arterial roads," Journal of Traffic and Transportation Engineering, vol. 18, no. 3, pp. 157-166, 2018.

[29] P. Ryus, M. Vandehey, L. Elefteriadou, R. G. Dowling, and B. S. Ostrom, Highway Capacity Manual, Transportation Research Board, Washington, DC, USA, 2010.

[30] F. Viti, The dynamics and the uncertainty of delays at signals, Doctoral thesis, TRAIL Research School, Delft, Netherlands, 2006.

[31] A. A. Markov, "Extension of the limit theorems of probability theory to a sum of variables connected in a chain," Dynamic Probabilistic Systems, vol. 1, pp. 552-577, 1971.

[32] China MoHaU-RDotPsRo, Code for Design of Urban Road Engineering, China Architecture and Building Press, Beijing, China, 2012. 